Pacific Journal of Mathematics

ON ARITHMETIC PROPERTIES OF COEFFICIENTS OF 


\title{
ON ARITHMETIC PROPERTIES OF COEFFICIENTS OF RATIONAL FUNCTIONS
}

\author{
DAVID G. CANTOR
}

\begin{abstract}
The purpose of this note is to prove the following generalization of a result of Polya:
\end{abstract}

Theorem. Let $\left\{a_{n}\right\}$ be a sequence of algebraic integers, and $f$ a nonzero polynomial with complex coefficients. If $\sum_{n=0}^{\infty} f(n) a_{n} z^{n}$ is a rational function, then so is $\sum_{n=0}^{\infty} a_{n} z^{n}$.

Polya [3] has proved that if $\sum_{n=0}^{\infty} n a_{n} z^{n}$ is a rational function, then so is $\sum_{n=0}^{\infty} a_{n} z^{n}$. It follows immediately from Polya's result that if $k$ is a rational integer and $\sum_{n=0}^{\infty}(n-k) a_{n} z^{n}$ is a rational function, then so is $\sum_{n=0}^{\infty} a_{n} z^{n}$. It is then easy to prove inductively, that if $f$ is a polynomial with complex coefficients, all of whose roots are rational integers, and if $\sum_{n=0}^{\infty} f(n) a_{n} z^{n}$ is a rational function, then so is $\sum_{n=0}^{\infty} a_{n} z^{n}$.

Suppose $K$ is an algebraic number field and $A \subset K$ is an ideal. If $\alpha$ and $\beta$ are algebraic numbers in $K$, we say, as usual, that $\alpha \equiv \beta(A)$, if there exists a rational integer $r$, relatively prime to $A$, such that $r \alpha$ and $r \beta$ are algebraic integers and $(r \alpha-r \beta) \in A$. We say that $A$ divides the numerator (denominator) of $\alpha$ if $\alpha \equiv 0(A) \quad((1 / \alpha) \equiv 0(A))$. We denote the norm of the ideal $A$ by $N m A$.

LEMMA 1. Let $K$ be an algebraic number field and $\alpha \in K$ an algebraic number. Then the set of those prime ideals of $K$ which divide the numerator of some element of the sequence $\{k-\alpha: k=$ $1,2,3, \cdots\}$ is infinite.

Proof. Suppose $n$ is a rational integer such that $n \alpha$ is an algebraic integer, and suppose $P_{1}, P_{2}, \cdots, P_{r}$ are the only prime ideal divisors of the sequence $\{n k-n \alpha: k=1,2,3, \cdots\}$. Now $N m(n k-n \alpha)$ is a nonconstant polynomial $g(k)$ with rational integral coefficients. Hence for each rational integer $k$, there exist rational integers $s_{1}, s_{2}, \cdots, s_{r}$ such that $g(k)=\mp \prod_{i=1}^{r}\left(N m P_{i}\right)^{s_{i}}$. Thus there are only finitely many rational primes which divide some element of the sequence $\{g(k): k=1,2,3, \cdots\}$. But this is false [2, p. 82].

REMARK. A less elementary proof of Lemma 1 is obtained by observing that if $P$ is a prime ideal with residue class degree 1 , and not dividing the denominator of $\alpha$, then there exists a rational integer

Received August 13, 1963. 
$n$ such that $n \equiv \alpha(P)$; since the set of such prime ideals has Dirichlet density 1, among all prime ideals, there are infinitely many of them.

LeMma 2. Suppose $\left\{a_{n}\right\}$ is a sequence of algebraic integers and $\alpha$ is an algebraic number. If $\sum_{n=0}^{\infty}(n-\alpha) a_{n} z^{n}$ is a rational function then so is $\sum_{n=0}^{\infty} a_{n} z^{n}$.

Proof. Since $\sum_{n=0}^{\infty}(n-\alpha) a_{n} z^{n}$ is a rational function, there exist distinct nonzero algebraic numbers $\theta_{1}, \theta_{2}, \cdots, \theta_{m}$ and polynomials with algebraic coefficients $\lambda_{1}, \lambda_{2}, \cdots, \lambda_{m}$ such that

$$
(n-\alpha) a_{n}=\sum_{i=1}^{m} \lambda_{i}(n) \theta_{i}^{n},
$$

for all $n \geqq n_{0}$, where $n_{0}$ is a rational integer. By replacing the sequence $\left\{a_{n}\right\}$ by the sequence $\left\{a_{n+n_{0}}\right\}$ if necessary, we may assume that (1) holds for all $n \geqq 0$. Let $K$ be an algebraic number field which contains $\alpha$, the coefficients of the $\lambda_{i}$, and the $\theta_{i}$. Choose a rational integer $k$ and a prime ideal $P \subset K$ such that $P$ divides the numerator of $k-\alpha$ and does not divide the numerator or denominator of $\alpha$, the $\theta_{i}$, the differences $\left(\theta_{i}-\theta_{j}\right)(i \neq j)$, and the coefficients of the $\lambda_{i}$; by Lemma 1 , there are infinitely many choices for the prime ideal $P$. Suppose that $N m P=p^{f}$ where $p$ is a rational prime. We substitute $n=k+j p^{f}$ in (1), where $j$ is a rational integer:

$$
\left(k+j p^{\jmath}-\alpha\right) a_{n}=\sum_{i=1}^{m} \lambda_{i}\left(k+j p^{\jmath}\right) \theta_{i}^{k+j p f} .
$$

Since $p^{f} \equiv 0(P)$ and $k \equiv \alpha(P)$, we obtain

$$
0 \equiv \sum_{i=1}^{m} \lambda_{i}(\alpha) \theta_{i}^{k} \theta_{i}^{j p f}(P) \text {. }
$$

But $\theta_{i}^{j p^{f}} \equiv \theta_{i}^{j}(P)$, hence

$$
\sum_{i=1}^{m} \lambda_{i}(\alpha) \theta_{i}^{k+j} \equiv 0(P)
$$

The $m$ equations obtained from (2) by successively substituting $j=$ $0,1,2, \cdots, m-1$ are linear in the $\lambda_{i}(\alpha)$ and have as determinant $\prod_{i=1}^{m} \theta_{i}^{k}$ times the Vandermonde determinant $\operatorname{det}\left\|\theta_{i}^{j}\right\|, 1 \leqq i \leqq m, 0 \leqq$ $j \leqq m-1$, which is not $\equiv 0(P)$, since $P$ does not divide any of the $\theta_{i}$ or the differences $\left(\theta_{i}-\theta_{j}\right)(i \neq j)$. Hence

$$
\lambda_{i}(\alpha) \equiv 0(P), 1 \leqq i \leqq m .
$$

By Lemma 1, (3) is true for infinitely many prime ideals $P$, hence $\lambda_{i}(\alpha)=0,1 \leqq i \leqq m$. It follows that the polynomials $\lambda_{i}(n)$ are divis- 
ible by $n-\alpha$. Put $\mu_{i}(n)=\lambda_{i}(n) /(n-\alpha) ; \mu_{i}(n)$ is a polynomial with algebraic coefficients. By (1)

$$
a_{n}=\sum_{i=1}^{m} \mu_{i}(n) \theta_{i}^{n}
$$

Thus $\sum_{n=0}^{\infty} a_{n} z^{n}$ is a rational function.

LEMMA 3. Suppose $\left\{a_{n}\right\}$ is a sequence of algebraic numbers and $f$ is a nonzero polynomial with complex coefficients. If $\sum_{n=0}^{\infty} f(n) a_{n} z^{n}$ is a rational function, then there exists a nonzero polynomial $g$ with algebraic coefficients snch that $\sum_{n=0}^{\infty} g(n) a_{n} z^{n}$ is a rational function.

Proof. There exist distinct nonzero complex numbers $\theta_{1}, \theta_{2}, \cdots, \theta_{m}$ and nonzero polynomials with complex coefficients $\lambda_{1}, \lambda_{2}, \cdots, \lambda_{m}$ such that

$$
f(n) a_{n}=\sum_{i=1}^{m} \lambda_{i}(n) \theta_{i}^{n},
$$

for all large $n$. Without loss of generality, we may assume that (4) holds for all $n \geqq 0$. In what follows, all fields are considered as subfields of the field of complex numbers. Denote by $\Omega$ the field of algebraic numbers, and by $L$ the smallest field which contains $\Omega$, the $\theta_{i}$, and all of the coefficients of the polynomials $f, \lambda_{1}, \lambda_{2}, \cdots, \lambda_{m}$.

Since $L$ is finitely generated over $\Omega$, it has a finite transcendence basis $x_{1}, x_{2}, \cdots, x_{r}$. Each of the $\theta_{i}$, the coefficients of the $\lambda_{i}$, and the coefficients of $f$ satisfies an irreducible polynomial equation whose coefficients are elements of $\Omega\left[x_{1}, x_{2}, \cdots, x_{r}\right]$. Let $h_{1}, h_{2}, \cdots, h_{s}$ be all of the nonzero coefficients of these polynomials; $h_{1}, h_{2}, \cdots, h_{s}$ are polynomials in $x_{1}, x_{2}, \cdots, x_{r}$ with coefficients in $\Omega$. Since there are only finitely many such polynomials, there exist algebraic numbers $\xi_{1}, \xi_{2}, \cdots, \xi_{r}$ such that $h\left(\xi_{1}, \xi_{2}, \cdots, \xi_{r}\right) \neq 0,1 \leqq i \leqq s$. The map $x_{i} \rightarrow \xi_{i}$ gives rise to a homomorphism of the ring $\Omega\left[x_{1}, x_{2}, \cdots, x_{r}\right]$ onto $\Omega$, which is the identity on $\Omega$. By the extension of place theorem $[1, \mathrm{p} .8]$, this homomorphism can be extended to a place $\varphi: L \rightarrow \Omega$, which is the identity on $\Omega$. If $\alpha \in L$, we denote by $\bar{\alpha}$ the image of $\alpha$ under $\varphi$ and if $b$ is a polynomial, $b(n)=\sum_{i=1}^{t} b_{i} n^{i}$ with coefficients $b_{i} \in L$, we denote by $\bar{b}$ the polynomial with $\bar{b}(n)=\sum_{i=1}^{t} \bar{b}_{i} n^{i}$. The $\theta_{i}$ and the coefficients of $f, \lambda_{1}, \lambda_{2}, \cdots, \lambda_{m}$ satisfy nonconstant polynomials $g_{1}, g_{2}, \cdots, g_{v}$ with nonzero constant term; the nonzero coefficients of these polynomials are the $h_{j}$. Under the place $\varphi$ the $h_{j}$ go into finite nonzero algebraic numbers $\bar{h}_{j}$. Hence the polynomial $\bar{g}_{k}$ has the same degree as $g_{k}$, all of its terms are finite, and its constant term is not zero $(1 \leqq k \leqq v)$. The $\bar{\theta}_{i}$ and the coefficients of $\bar{f}, \bar{\lambda}_{1}, \bar{\lambda}_{2}, \cdots, \bar{\lambda}_{r}$ are roots of these poly- 
nomials; hence the $\bar{\theta}_{i}$ are finite, nonzero algebraic numbers, and the $\bar{f}, \bar{\lambda}_{1}, \bar{\lambda}_{2}, \cdots, \bar{\lambda}_{m}$ are nonzero polynomials, with finite, algebraic coefficients. Applying the place $\varphi$ to both terms in (4), and putting $\bar{f}=g$, yields, since $\bar{a}_{n}=a_{n}$

$$
g(n) a_{n}=\sum_{i=1}^{m} \bar{\lambda}_{i}(n) \bar{\theta}_{i}^{n}
$$

Hence

$$
\sum_{n=0}^{\infty} g(n) a_{n} z^{n}=\sum_{n=0}^{\infty} \sum_{i=1}^{m} \bar{\lambda}_{i}(n) \bar{\theta}_{i}^{n} z^{n}
$$

is a rational function, and $g$ is a nonzero polynomial with algebraic coefficients.

Proof of theorem. By Lemma 3, we may assume that $f$ has algebraic integer coefficients. Let $\alpha$ be a root of $f$ and $g(n)=f(n) /(n-\alpha)$; by the lemma of Gause, $g(n)$ is a polynomial with algebraically integral coefficients. Put $b_{n}=g(n) a_{n} ;\left\{b_{n}\right\}$ is a sequence of algebraic integers and $\sum_{n=0}^{\infty}(n-\alpha) b_{n} z^{n}$ is a rational function. By Lemma 2 , so is $\sum_{n=0}^{\infty} b_{n} z^{n}$. Proceeding inductively, on the degree of $f$, we see that $\sum_{n=0}^{\infty} a_{n} z^{n}$ is a rational function.

REMARK. By the Remark following Lemma 1, one can replace, in the theorem, the requirement that the $a_{n}$ be integers, by the requirement that the set of prime ideal divisors of the denominators of the $a_{n}$ has Dirichlet density less than 1 among all prime ideals.

Let $f(z)=\sum_{n=0}^{\infty} a_{n} z^{n}$, where the $\alpha_{n}$ are rational integers. Polya's theorem then asserts that if $f^{\prime}(z)$ is a rational function, so is $f(z)$. The corresponding assertion of our generalization of Polya's theorem is: Let $f(z)=\sum_{n=0}^{\infty} a_{n} z^{n}$ be a power series with algebraically integral coefficients. If there exists a nonzero differential operator $L$, of the form $L=$ $\sum_{i=0}^{r} c_{i}(z d / d z)^{i}$ ( $c_{i}$ complex numbers), such that $L f$ is a rational function, then so is $f(z)$.

\section{REFERENCES}

1. S. Lang, Introduction to algebraic geometry, New York, 1958.

2. T. Nagell, Introduction to number theory, New York, 1958.

3. G. Polya, Arithmetische Eigenschaften der Reihenentwicklungen, J. Reine u. angew. Math., 151 (1921), 1-31. 


\section{PACIFIC JOURNAL OF MATHEMATICS}

\section{EDITORS}

H. Samelson

Stanford University

Stanford, California

R. M. Blumenthal

University of Washington

Seattle, Washington 98105
J. Dugundu

University of Southern California Los Angeles, California 90007

*Richard Arens

University of California

Los Angeles, California 90024

\section{ASSOCIATE EDITORS}
E. F. BECKENBACH
B. H. NeumanN
F. WOLF
K. YOSIDA

\section{SUPPORTING INSTITUTIONS}

UNIVERSITY OF BRITISH COLUMBIA CALIFORNIA INSTITUTE OF TECHNOLOGY UNIVERSITY OF CALIFORNIA MONTANA STATE UNIVERSITY

UNIVERSITY OF NEVADA

NEW MEXICO STATE UNIVERSITY

OREGON STATE UNIVERSITY

UNIVERSITY OF OREGON

OSAKA UNIVERSITY

UNIVERSITY OF SOUTHERN CALIFORNIA
STANFORD UNIVERSITY

UNIVERSITY OF TOKYO

UNIVERSITY OF UTAH

WASHINGTON STATE UNIVERSITY

UNIVERSITY OF WASHINGTON

AMERICAN MATHEMATICAL SOCIETY CALIFORNIA RESEARCH CORPORATION SPACE TECHNOLOGY LABORATORIES NAVAL ORDNANCE TEST STATION 


\section{Pacific Journal of Mathematics}

\section{Vol. 15, No. $1 \quad$ September, 1965}

Donald Charles Benson, Unimodular solutions of infinite systems of linear

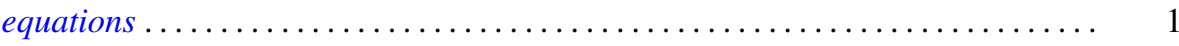

Richard Earl Block, Transitive groups of collineations on certain designs . . . . . . 13

Barry William Boehm, Existence of best rational Tchebycheff approximations .... . 19

Joseph Patrick Brannen, A note on Hausdorff's summation methods . . . . . . . . . . 29

Dennison Robert Brown, Topological semilattices on the two-cell ............ 35

Peter Southcott Bullen, Some inequalities for symmetric means . . . . . . . . . . 47

David Geoffrey Cantor, On arithmetic properties of coefficients of rational

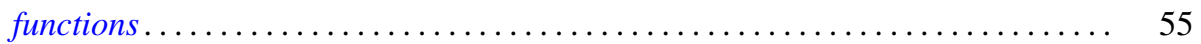

Luther Elic Claborn, Dedekind domains and rings of quotients . . . . . . . . . 59

Allan Clark, Homotopy commutativity and the Moore spectral sequence ........ 65

Allen Devinatz, The asymptotic nature of the solutions of certain linear systems of

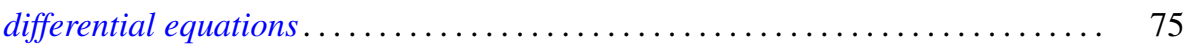

Robert E. Edwards, Approximation by convolutions ................... 85

Theodore William Gamelin, Decomposition theorems for Fredholm operators . . . . . 97

Edmond E. Granirer, On the invariant mean on topological semigroups and on

topological groups .................................. 107

Noel Justin Hicks, Closed vector fields . . . . . . . . . . . . . . . . . . . 141

Charles Ray Hobby and Ronald Pyke, Doubly stochastic operators obtained from

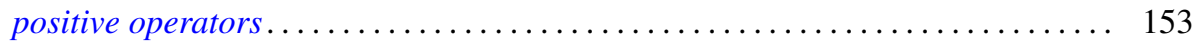

Robert Franklin Jolly, Concerning periodic subadditive functions . . . . . . . . . 159

Tosio Kato, Wave operators and unitary equivalence . . . . . . . . . . . . . . 171

Paul Katz and Ernst Gabor Straus, Infinite sums in algebraic structures . . . . . . . 181

Herbert Frederick Kreimer, Jr., On an extension of the Picard-Vessiot theory ...... 191

Radha Govinda Laha and Eugene Lukacs, On a linear form whose distribution is

identical with that of a monomial ......................... 207

Donald A. Ludwig, Singularities of superpositions of distributions . . . . . . . . . 215

Albert W. Marshall and Ingram Olkin, Norms and inequalities for condition

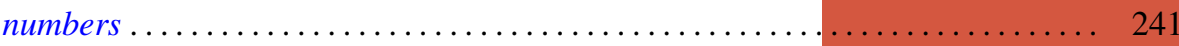

Horace Yomishi Mochizuki, Finitistic global dimension for rings . . . . . . . . . . 249

Robert Harvey Oehmke and Reuben Sandler, The collineation groups of division

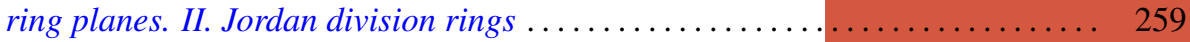

George H. Orland, On non-convex polyhedral surfaces in $E^{3} \ldots \ldots \ldots \ldots \ldots \ldots \ldots 267$

Theodore G. Ostrom, Collineation groups of semi-translation planes . . . . . . . . 273

Arthur Argyle Sagle, On anti-commutative algebras and general Lie triple

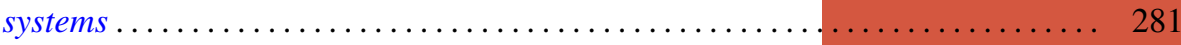

Laurent Siebenmann, A characterization of free projective planes . . . . . . . . . 293

Edward Silverman, Simple areas.................................. 299

James McLean Sloss, Chebyshev approximation to zero .................. 305

Robert S. Strichartz, Isometric isomorphisms of measure algebras . . . . . . . . . 315

Richard Joseph Turyn, Character sums and difference sets . . . . . . . . . . . . 319

L. E. Ward, Concerning Koch's theorem on the existence of arcs . . . . . . . . . . 347

Israel Zuckerman, A new measure of a partial differential field extension ......... 357 